\title{
VIRTUAL LABORATORIES AND VIRTUAL ORGANIZATIONS SUPPORTING BIOSCIENCES
}

\author{
E. C. Kaletas, H. Afsarmanesh, L. O. Hertzberger \\ University of Amsterdam, Informatics Institute \\ (kaletas, hamideh, bob)@science.uva.nl
}

THE NETHERLANDS

\begin{abstract}
Emergence of advanced complex applications in applied sciences poses many challenges to both the scientists and the developers of tools and mechanisms for their supporting infrastructure. Although technological advances in highthroughput / high-performance technologies provide the base, tackling the challenges in this area require long-term multi-disciplinary efforts between scientists and computer science experts. One such multi-disciplinary effort is started in bio-informatics, considering the growing complexity of emerging experiments and data processing requirements in biosciences domain. Among others, challenges in bio-sciences include designing experiments that utilize advanced technologies for storage, manipulation, and integration/interlinking of vast amounts of data produced by experiments, analysis of results on highperformance computers, and accessing the highly heterogeneous information that resides at geographically scattered centers. In this paper, we propose an "enhancement environment" for a virtual laboratory approach, supporting scientists to overcome the above problems and assisting them during their experimentation. Furthermore, we describe how this enhancement environment can be extended, applying the virtual organizations concept and infrastructures, to support the collaborative experiments among diverse, heterogeneous and autonomous centers in the biosciences domain'.
\end{abstract}

\section{INTRODUCTION}

Systematic growth of research efforts in experimental sciences has resulted in gathering vast amounts of data/information in a wide range of resources distributed all around the world. In many cases, the end-users accessing the data/information are also geographically distributed. In biosciences for instance, new generation of automated high-throughput experimental technologies has paved the way for orders of magnitude larger and more complex experiments; leading to exponential growth in size of genome databases. At the present rate of growth, the SWISS-PROT (Bishop, 1999) database for instance, will double in size every 40 months and the nucleotide databases will double in size almost every 14 months (Williams, 1999). During the year 2000, every day over two million bases were deposited into GenBank (Bishop, 1999), and the bio-informatics companies have already generated and store terabytes of genomic data (Celera, 2001). 
Considering the complexity of biosciences domain and its emerging experimentations, the big challenge in fact falls into two areas of biology and computer science. In biology, scientists involved in these experimentations must both advance their models and mechanisms supporting the analysis of resulted information, and design innovative investigation approaches leading to discoveries. In computer science, the main challenges fall in the areas of 1) data/information handling, management, and analysis, 2) high performance computation infrastructures, 3) interoperation and collaboration infrastructures, and 4) development of enhancement environments to support scientists with their complex activities.

With the achieved advances so far in biosciences, the human genome is mostly sequenced, several metabolic pathways are identified, and expression profiles have been generated for many genes, among other, opening the doors to bigger possibilities for new discoveries and advanced research, through the "extraction of knowledge" from the scattered data that is already collected. Achieving such advanced experimentations, either being performed by individual scientists or in collaboration with other centers, primarily constitute "large processes" involving many sub-steps. Namely, several distributed, heterogeneous, and autonomous sites need to be inter-linked, and perhaps co-work towards the achievement of a common experiment. High performance computing environments are necessary for mining and extracting this knowledge. Advanced interoperation/collaboration infrastructures as well as federated cooperative information management is required to access, retrieve and inter-link data from diverse, heterogeneous, and autonomous databases and other kinds of data resources. Clearly, this is an involved process that both requires, and can benefit from specialized "enhancement environments" providing models, mechanisms, and tools, enabling scientists and centers with their complex and relatively long experimentation process. The enhancement environments developed by computer scientists facilitate the wide variety of needed functionalities.

Considering different areas within biosciences, research has resulted a large number and range of "heterogeneous" genome databases. The heterogeneity is mainly due to: (1) the wide variety of types of genomic information, (2) various representations/formats designed for the stored data, and (3) various access mechanisms to data. In the latest compilation of key high-quality biological databases all around the world (Baxevanis, 2001), 281 databases are listed, classified in 18 different categories based on their biological content. Every such database is provided and supported by an independent and "autonomous" center. Out of these databases, 55 were added only since January 2000 . Furthermore, many centers store this diverse data/information in flat files, while each defining its own specific datastructure and data-format. Others use different database management systems (DBMS) to store data, but even if the same DBMS is used by two centers, there are enormous differences in their design of data representation (data models/data formats). Querying capabilities and data retrieval interfaces also change from one data source to another. The heterogeneity problem grows further with the relatively new types of biosciences information, for instance the "gene expression" data, where even the consensus on defining the required minimum information set to be stored for gene expression is still under study (MGED, 2001). 
Another effect of advances in technology is that the new generation experimental technologies are becoming more complex in terms of the required instruments and infrastructure. On one hand, to effectively utilize a new instrument or advanced computing environments, wide experience becomes the key requirement. On the other hand, increasing costs of acquiring and operating such instruments force institutions to think twice before procuring and installing the instrument. An environment that allows sharing the skills as well as resources can certainly support scientists in their experiments

Clearly there is a pressing need to assist researchers with enhancement environments in conducting their complex scientific experiments. One such enhancement environment is a "Virtual-Laboratory" (Afsarmanesh et al., 2001a, DOE, 2000) which can support scientists by the provision of proper hardware and software assisting infrastructure, to systematically define and carry out their experiments. Another such assisting environment is a "Virtual-Organization" (Camarinha-Matos and Afsarmanesh, 1999) which supports independent sites collaborating on one common project by providing proper infrastructure for co-ordination of the joint distributed activities, and their secure and authorized exchange of information. Sharing expensive resources (e.g. for management and publishing of information, for provision of tools such as simulation and visualization tools, and for accessing to complex instruments) among various individuals or research centres via virtuallaboratories, and sharing skills, distributing the tasks, and integrating required information among enterprises via virtual-organisations, among other benefits, reduces the experimentation costs while at the same time enables scientists to focus more on their experiments' definitions and complexities (Afsarmanesh et al., 2001b).

The rest of the paper is organized as follows: As motivation, Section 2 sketches a scenario case that illustrates the problems that a biologist faces when making a new micro-array experiment. In Section 3, we will present how the VLAM-G environment will help this biologist to overcome part of his problems. Section 4 describes how applying the VO concept to the biosciences can help a biologist to perform an advanced collaborative experiment.

\section{MOTIVATION - Complexity of Biosciences Experiments}

Consider the scenario where a biologist at a pharmaceutical company is working on a new drug. Let us assume this drug is targeting a specific disease, for which the responsible gene is already known. He wants to test whether the new drug is indeed preventing the activities of the target gene and under which conditions (e.g. concentration of the active materials) the drug performs more successfully. In order to investigate the activities of the target gene, the biologist needs to make a microarray experiment.

DNA micro-arrays allow genome-wide monitoring of changes in gene expression levels in response to some stimuli, for instance, the expression of a gene in response to certain drug. With the current technology of DNA micro-arrays, it is possible to study the characteristics of thousands of genes within a single experiment. 
To perform this complex study, the biologist first designs a micro-array experiment. In general, the steps in a micro-array experiment can be grouped into five major tasks; clone preparation, micro-array production, cDNA-probe preparation, hybridization, scanning and image analysis, and finally analysis of the results. An abstract overview of a micro-array experiment is given in Figure 1. However, depending on the experiment setup and on the already available biomaterial in the lab, some of these steps (or even tasks) can be skipped. In our case, we assume that the pharmaceutical company does not prepare the clones itself rather acquires them from another biotechnology company, while all other required biomaterial will be prepared in house. Thus, the experiment will consist of the steps involved in the remaining four tasks. Please notice here that the biologist should possess the necessary expertise about the procedure to follow (i.e. which steps are involved in each task) and the protocols to apply them (i.e. how each step is performed). Due to its complexity, once the steps to follow and how to perform them are defined, the experiment definition needs to be stored for future references.

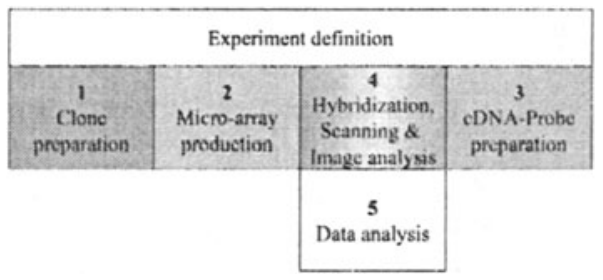

Figure 1 - Abstract overview of a micro-array experiment

During the actual experimentation phase, first the DNA clones obtained from the biotechnology company must be spotted on micro-array slides using a spotter device. Current spotters are capable of spotting up to 40,000 clones on a single micro-array slide. The DNA clones uniquely represent the genes of the organism under study. At this stage, it is extremely important to keep track of which clone is spotted on which micro-array slide and to which position in that slide, as well as the genes corresponding to the clones and the organism information. Meanwhile, samples from both untreated cells and cells treated with the drug should be prepared. Messenger RNA pools representing all expressed genes (active genes) in the cell types under investigation should be extracted from the samples, cDNA copies must be synthesized and labeled with (usually) either a red or green fluorescent dye. Each dye will represent a different cell type, e.g. red dye for drug treated cells. Once both the micro-arrays and cDNA-probes are ready, they are hybridized, that is, microarrays are treated with the two labeled cDNA probes. cDNAs will only bind to DNA clones on the micro-arrays that contain identical or highly similar nucleotide sequence. The unbound probes will be washed away, remaining only the probes bound to some clones on the micro-array. The hybridized micro-arrays are then scanned to read the level of fluorescence for each dye and each spot on the slide, producing high-resolution computer images. Analysis of the image will quantify the relative levels of gene expression for each gene represented on the micro-array, resulting in around 300,000 useful data points for human. Please notice that it is crucial to $\log$ the detailed conditions and results at each step during the experiment, since without this information it is impossible to interpret the experimental results. 
However, in order to see whether the developed drug indeed prevented the activities of the gene under study or not, the biologist has to analyze the experiment results. In our example, the biologist will cluster the expression results, in order to compare the target gene expression against the expression values of other known genes with similar activities. This comparison can also be extended to cover the results of the previous micro-array experiments. Please notice that depending on the available information about the drug and target gene, several micro-array experiments may be required, each using samples treated with the drug under certain specific conditions. In this case, information about all experiments has to be grouped to indicate the relation among them, and the analysis of the experiment results may include the usage of many software packages in combination, such as selforganizing maps for hierarchical clustering, or Pearson algorithm for statistical analysis. Only at this point, the biologist knows whether the target gene was expressed or suppressed after the drug treatment.

\section{VIRTUAL LABORATORY (VL) - The VLAM-G Project}

Clearly there is a need for advanced systems to assist researchers with conducting their emerging complex scientific experimentations. Modern advances in the IT area and new approaches such as Virtual Laboratories (VL) (DOE, 2000) prove to be successful for tackling this challenge. The ICES/KIS-II project VLAM-G (Gridbased Virtual Laboratory Amsterdam) (Afsarmanesh et al., 2001a) of the University of Amsterdam (UvA) aims at the design and development of an open, flexible, scalable, and configurable framework providing necessary GRID-based hardware and software enabling scientists and engineers in different areas of research to work on their problems via experimentation, while making optimum use of the modern Information Technology. The VLAM-G provides a distributed high performance computing and communication VL infrastructure with advanced information management functionalities, addressing in specific the experimentation requirements in the scientific domains of biology, physics, and systems engineering. As such, access to physically distributed data and processes among many sites in the virtual laboratory, that is necessary for the achievement of complex experimentations, will be totally transparent to the scientists, giving them the image of working in a single physical laboratory.

An architectural overview of the VLAM-G is given in Figure 2. The Web-based portal and workbench interface together with the modular design of the VLAM-G architecture provides a uniform environment for all experiments, and makes it possible to attach a wide range of software tools to the laboratory; from basic tools such as simulation, visualization, data storage / manipulation to advanced facilities like: remote controlling of devices (in COMCOL), visualization in a virtual reality environment (in VISE), and federated advanced information management (in VIMCO). VLAM-G RTS is a Grid-based run-time environment providing distributed processing, networking and resource management facilities to VLAM-G applications. In VLAM-G, modularity is the key to scalability and openness, also to support the inter-disciplinary research. The VLAM-G solves many technical problems that scientists face, hence enabling them to focus better on their 
experiments, while simultaneously reducing the costs of experimentation by sharing the expensive resources among them.

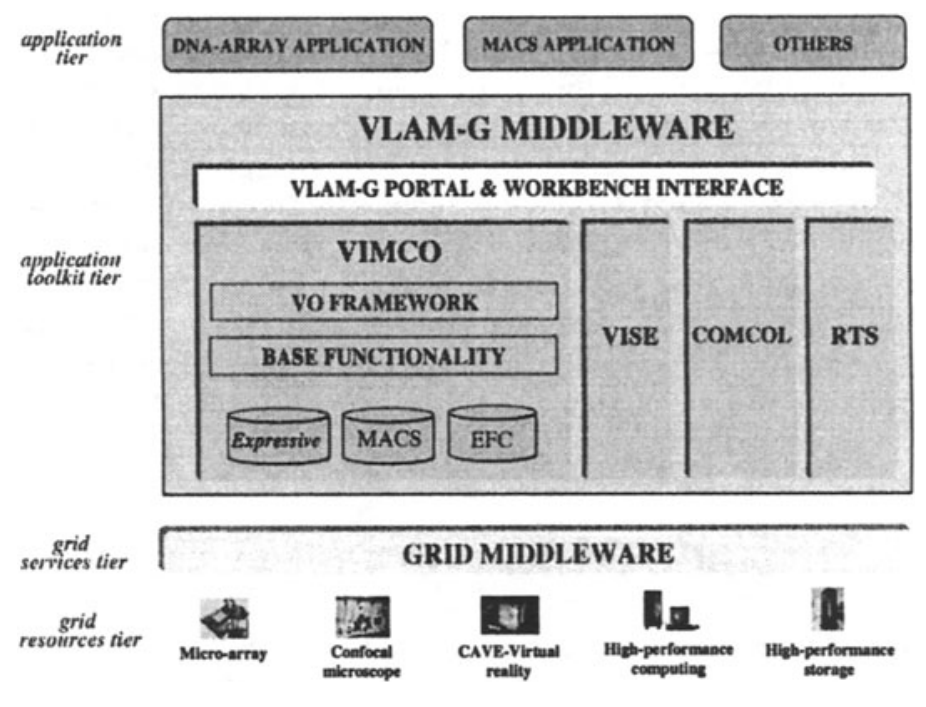

Figure 2 - VLAM-G architectural overview

In order to better illustrate the functionality offered by VLAM-G, let us see how it can help our biologist in his micro-array experiment.

\section{Designing the experiment:}

The VLAM-G portal and workbench provides a user-friendly and uniform environment to the scientists, where they can define and execute their experiments.

For each of the VLAM-G applications, a PFT (process-flow template) is defined and stored in VIMCO to guide the experimenter throughout the experimental procedure. In our example, the biologist is presented a DNA micro-array PFT; which contains the steps involved in a typical micro-array experiment. He will go through this template step by step, and enter the required information for each step into the fill-in forms displayed. Every form corresponds to a specific step in the experiment, and captures the information required for that step. For instance, the molarity of the solvent used in hybridization, resolution parameter used for the image analysis software, or comments of the biologist on the quality of the clones obtained from the biotechnology company. If the biologist has already the experience in micro-arrays, he can customize the micro-array PFT and define his own template, which he can save to use in the future as well.

\section{Performing the experiment:}

The VLAM-G functionality for data analysis, data manipulation, visualization, etc. is provided as software modules. The standardized interface of these modules is the key for software interoperability in VLAM-G, allowing users to attach a number of modules to each other to perform a specific task. 
When one of the experiment steps in the PFT involves, for instance, the usage of a device attached to the VLAM-G, or high-performance distributed computing, the scientist is presented a screen, which contains the available software modules. For example, in the micro-array scenario, the analysis of the scanned micro-array images requires a certain amount of computational power and storage. In this step, the biologist selects the most suitable analysis software among the available biology related software modules, and attach it to the modules that reads the images from the archive and stores the analysis results in the gene expression database. When defined, the workflow (software modules connected to each other) will be first saved and then executed by the VLAM-G Run Time System (RTS).

VLAM-G RTS is Grid based, and VLAM-G can be seen as middleware between the Grid tier and the applications tier. The VLAM-G RTS executes the modules in the workflow on the Grid in a distributed manner using the Globus toolkit (Foster and Kesselman, 1998). This allows the users to specify the computational, networking, and storage requirements for the execution of their modules and acquire the required resources from a distributed pool of available resources.

Furthermore, an intelligent assistant agent will provide scientists some statistical information, such as information about similar tools, most-used software modules, some run-time statistics about a module, etc.

\section{Analysis of the experiment results:}

In order to extract the knowledge and have better insights on the problem under study, the data sets obtained after the experiment needs to be analyzed. The analysis can involve among others utilization of high computational power, high-speed networks, storage facilities, and visualization tools. The VLAM-G environment provides its users the hardware and software facilities to support the analysis, such as Grid-based distributed computing farms connected to each other with gigabit networks, high capacity archiving systems and application databases for information storage, and simulation and virtual reality environments. Scientists can make use of these facilities in the same way that they execute their experiments; define a workflow for the analysis of the result data sets and submit the workflow to the RTS to be executed on the Grid.

In the micro-array example, the biologist will be able to choose the software modules to use in his workflow to analyze his expression result set. The analysis will first read the current experiment result set from the expression database, and perform a query on the database to retrieve the results of all experiments performed in the past for the same drug and for the same target gene. Then the current results set will be compared to the others to find any common expression behavior. Finally the comparison results will be displayed. Now assume that there are two analysis tools available for such a comparison, one performing the comparison in a sequential way on the same machine, the other distributing the result sets on a cluster and compare in parallel. The biologist will select the second module; connect the database module as input to this analysis module to read the data sets from the database, and a visualization and another database module as output to the analysis module to display the comparison results and store in the database. Alternatively, he can modify the parameters of the analysis module, display the results, and repeat the analysis until a better result is obtained, and store the results in the database. 
Information management:

During the micro-array experiment, not only the experiment results, but also the information about the experiment steps must be stored. Thus, the database model should be capable of capturing all possible annotations about an experiment that a scientist can define so that the experiment results can be compared with both local data sets and with other results made available by external scientific centers. In this direction, after studying the micro-array experiment in depth, a large set of aspects/entities common to DNA-array experiments were identified, and EEDM (Experimentation Environment Data Model), a base meta-data model for representation of experimental data (Kaletas et al., 2001), was designed. Based on the EEDM data model, EXPRESSIVE database is developed for gene expressions. EXPESSIVE aims at the storage and retrieval of both the steps and annotations involved in DNA micro-array experiments as well as the DNA micro-array experiment results (raw data and processed analysis results) through the VLAM-G.

VIMCO is the information management component of the VLAM-G. In VIMCO, in addition to the database modeling for experimental science domains, data manipulation mechanisms are also being developed. Several libraries supporting Web based and platform independent database access are provided. Furthermore, distributed and multi-threaded manipulation of data for multi-user access to VLAM-G environment, and XML-based export, import and data/information exchange facilities are being developed. The design of the microarray experiment, its annotations, resulting images and quantified expression values, and analysis results are stored in Expressive and high-capacity archives using the information management facilities provided by VIMCO.

Besides the EXPRESSIVE database focused on biology domain, two other databases are developed within the VLAM-G, namely MACS for the material analysis studies in physics, and EFC for traffic engineering. In addition to the validation of the Virtual Laboratory approach, these three specific applications provide and test the possibility of multi-disciplinary studies and experimentations. For instance, the proteomics studies will in fact require a cross-reference between the EXPRESSIVE and MACS databases. The information management aspects of VLAM-G are designed and developed by the Co-Operative Information Management (CO-IM) group of the University of Amsterdam (CO-IM).

\section{VIRTUAL ORGANIZATION/LABORATORY (VO/VL)}

As can be seen from the example scenario given above, VLAM-G is invaluable to help scientists during their experimentation. However, further extensions are required by many of the scientific applications of today, supporting the interoperation and collaboration among diverse heterogeneous and autonomous organizations. In this section, we will extend and use our micro-array example scenario to illustrate these required advanced functionalities, describing a VO-based solution.

In our example scenario, the biologist could successfully determine the expression pattern of the target gene in the drug-treated cells. Now we assume that the drug indeed prevents the activities of the gene. However, in order to turn the drug into a product, some more steps have to be taken; for instance, a BLAST search 
to find genes with similar sequences (to learn more about similar genes), a location map to find the location of the gene on the chromosome (to see if there is a disease associated with that chromosomal location), or a pathway search to find in which metabolic pathways the genes whose gene expressions have changed play a role (to see any side effects of the drug on those genes). Other information that needs to be collected includes the clones information from the biotechnology company, and chemical compounds information to check whether the compound used in the drug already exists (for patent issues). This information retrieval means accessing several databases one by one usually via some specific Web pages, browse the information which is in a different format in each database, combining the relevant information from these databases, and repeat this search possibly many times. (Finally, the results of all these searches must be stored). It might be the case that appropriate privileges are required for some proprietary databases, such as the clone database of the biotechnology company or the chemical compounds database.

Moreover, a full-fledged micro-array lab with spotter, scanner, slide processor, etc. is an expensive facility. It is a common practice that institutes and companies share the laboratory facilities. Hence, we can safely assume that our pharmaceutical company does not have a micro-array facility in house. For the experiment, the micro-array lab of a university might be used, which is Grid enabled hence accessible from VLAM-G. We can also assume that the computational facilities required for the analysis will be provided by the VLAM-G.

In order to develop the drug, the pharmaceutical company for instance needs to cooperate with the university to access the micro-array lab, with the biotechnology company for the clones and information, and with the chemical compounds company for the drug ingredients. This cooperation will help the micro-array lab to improve the lab facilities, and support the biotechnology and chemical compounds companies to continue with their research to provide better products. It is clear that cooperation among the institutes and companies each providing a resource is beneficial for each participant of the cooperation.

However, in order to properly support cooperation among the partners, rules of cooperation must be defined and mechanisms to preserve the autonomy of the independent companies must be provided. For instance, the pharmaceutical company and the micro-array lab must negotiate to define how many experiments can the company make in a week, who will provide which biomaterial to be used in the experiments, whether technician support is required from the lab or scientists from the company will make the experiments, how will be the form of payments, and how the security of the biomaterial used and experimental information will be provided. The answers to all these issues must be well defined and a contract must be prepared, which will be the legal base for cooperation between the company and the lab.

The Virtual Organizations concept suits properly addressing these issues. Extending the VLAM-G environment by applying the Virtual Organizations concept and infrastructures provides a stronger infrastructure to support the requirements of scientists in the biosciences domain. Having the necessary infrastructure, the cooperating institutes and companies in our example can establish a Virtual Organization (Figure 3). 


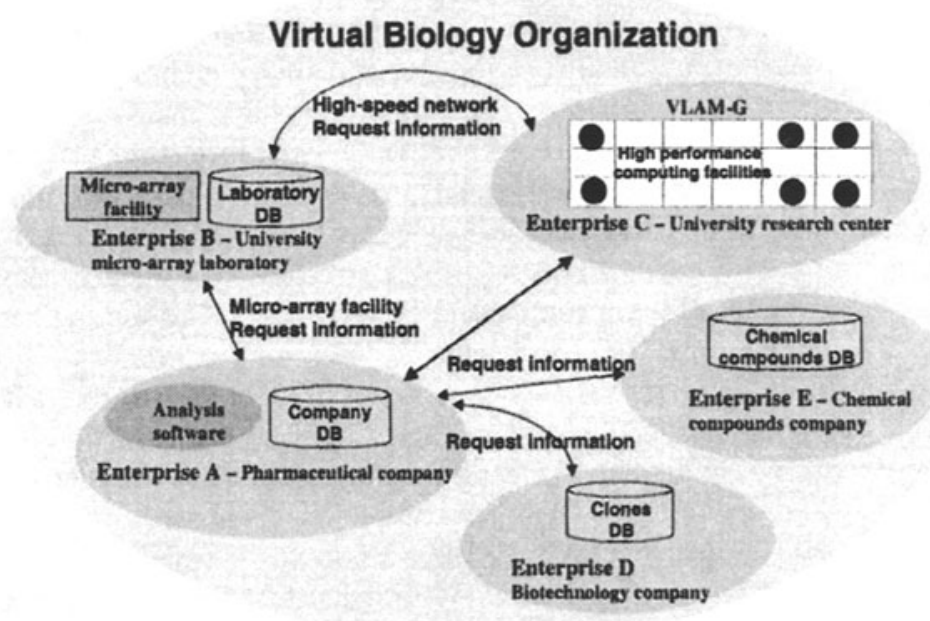

Figure 3 -Virtual Biology Organization

The necessary supporting infrastructure for VOs is being developed by the VIMCO component of VLAM-G. The infrastructure contains a catalogue and a federated framework. The catalogue stores information related to VOs, for instance, VO information (name, id, partners, contact, etc), and enterprise information (business area, services and data provided, contact information, etc). The federated framework will provide the mechanisms to define access rights on the available resources (data/information, hardware/software resources, etc) as a part of the VO contract and the enforcement of the contractual terms at access/execution time.

The federated framework, called ARCHIPEL, is currently at its design stage. The design of ARCHIPEL considers benefiting from the computation and communication facilities provided by the VLAM-G. Specifically, the distributed processing environment of the VLAM-G RTS, which is built on top the Grid, can support the inherently distributed nature of federated query processing (FQP) in ARCHIPEL. The current design of the ARCHIPEL Federated Query Processor is given in Figure 4. A mediator is developed for each (local and remote) data source accessible through the ARCHIPEL.

The dashed arrows in Figure 4-a represent the (global or local) queries and the solid arrows represent the query results (data). The ARCHIPEL FQP executes a global query as follows: When a global query arrives, the FQP generates a global query execution plan, in the form of a graph. The leaves of the graph (sq1, sq2, sq3 in Figure 4-a) represent the actual queries to be executed on the underlying local data sources. From the global query plan, the ARCHIPEL FQP decomposes the global query into sub-queries, namely queries on local or remote data sources. In Figure 4-a, two types of mediators are defined: Stationary Mediators and Grid Mediator. There exist one Stationary Mediators for each data source, which handles the communication with the data sources, i.e. performing the necessary translations and mappings of the queries and query results. These mediators run on the same node with the local data source. Grid Mediator, on the other hand, runs on a Grid- 
enabled node. After decomposing the global query into sub-queries, ARCHIPEL FQP checks whether any of the sub-queries involves a Grid-enabled data source. Since the FQP has the knowledge about which sub-query must go to which data source, as well as which mediator is responsible for which data source, it sends the sub-queries to the responsible mediators corresponding to the data sources. The mediators ultimately perform the necessary translations and required mappings to the data source in order to execute the sub-query, and return the query results back to the FQP. Finally, FQP merges the results of sub-queries into the global query result and sends it back to the user.

In Figure 4, ARCHIPEL FQP finds out that sub-queries 2 and 3 (sq2 \& sq3) needs to be executed remotely through the VL-MACS database and a Grid-enabled remote database, respectively; hence, it sends sq2 and sq3 to the Grid Mediator with sufficient information required about the data sources. As represented in Figure 4-b, Grid Mediator analyses the sub-queries, translates them into two jobs which will be executed by the VLAM-G RTS, and submits the jobs to the RTS together with the information about the data sources. The RTS follows a similar procedure that of the execution of experiment topologies, as described in Section 3.

After analysis of the received job requests, VLAM-G RTS instantiates two Mobile Mediator modules for the sub-queries, initializing them with sq2 and sq3, and using the Grid services, it sends the Mobile Mediators to the data source nodes for execution. The Mobile Mediators execute the sub-queries on the data sources, and send the query results to the Grid Mediator via the RTS.
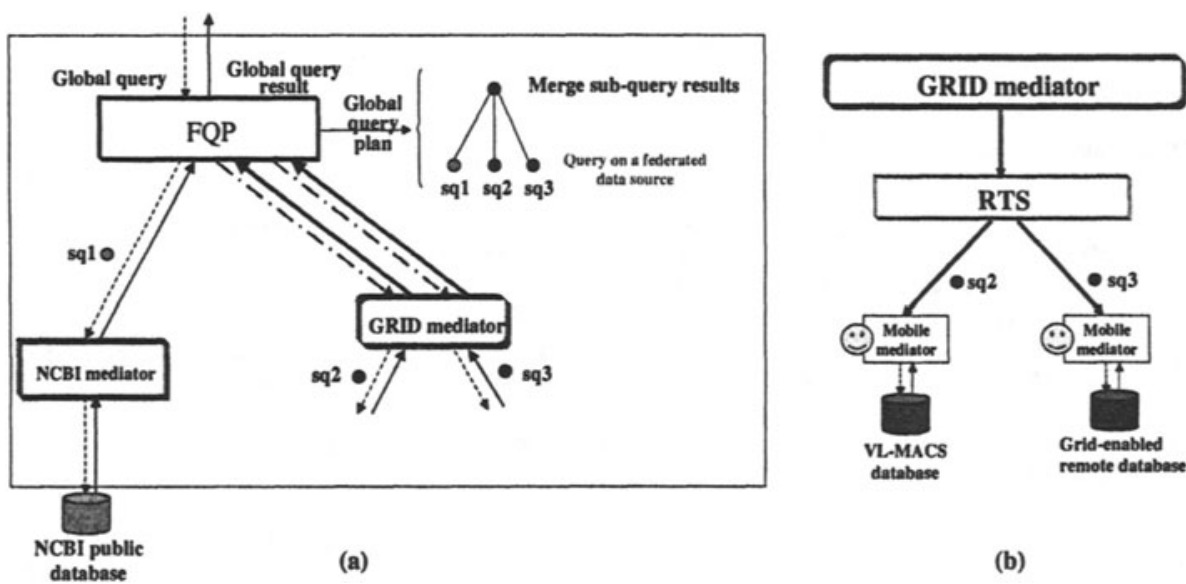

Figure 4 - (a) ARCHIPEL FQP and different types of mediators

(b) Grid Mediator using RTS and Mobile Mediators to execute sub-queries

Using the Grid Mediator and Mobile Mediators, ARCHIPEL FQP can fully utilize the distributed computing, networking, and job scheduling facilities of the VLAM-G RTS. Furthermore, application of Stationary Mediators make it possible to cover non-Grid data sources, making the ARCHIPEL FQP a generic query processor. 


\section{CONCLUSIONS}

New discoveries and emerging technologies in biosciences has resulted vast amounts of data/information and relatively complex experimental procedures. Challenges in biosciences require the application of advance solutions in both the biology and computer science areas. In this paper we have focused on the computer science related challenges, and presented the VLAM-G virtual laboratory solution and its extensions with the virtual organizations infrastructure as an enhancement environment. The VLAM-G helps biologists during experiment design via: uniform and friendly user interfaces, experiment execution and analysis of the results in a Grid-based distributed and high-performance infrastructure, and federated information management system to store the experiment definitions, experiment steps, and analysis results. Then we showed that this environment can be extended with the virtual organization concept, thus allowing the individual distributed sites (scientists, institutes, organizations, companies) to cooperate on solving a joint problem where the cooperation is based on a solid contract containing the terms of cooperation. Finally the design of a federated query processor supporting the described environment is presented, which fully utilizes the distributed computing, networking, and job scheduling facilities of the VLAM-G RTS.

\section{REFERENCES}

1. Afsarmanesh, H., Kaletas, E. C., Benabdelkader, A., Garita, C. and Hertzberger, L. O. (2001a) A reference architecture for scientific virtual laboratories, Future Generation Computer Systems, vol. 17.

2. Afsarmanesh, H., Kaletas, E. C. and Hertzberger, L. O. (2001b), The Potential of Grid, Virtual Laboratories and Virtual Organizations for Bio-sciences, in proceedings of SOFSEM 2001: 28th Conference on Current Trends in Theory and Practice of Informatics, Piest'any, Slovak Republic

3. Baxevanis, A. D. (2001) The Molecular Biology Database Collection: an updated compilation of biological database resources, Nucleic Acids Research, vol. 29.

4. Bishop, M. J. (Ed.) (1999) Genetics Databases, Academic Press, Hinxton, Cambridge.

5. Camarinha-Matos, L. M. and Afsarmanesh, H. (1999) The Virtual Enterprise Concept in Infrastructures for Virtual Enterprises - Networking Industrial Enterprises, (Eds, CamarinhaMatos, L. M. and Afsarmanesh, H.) Kluwer Academic, pp. 3-14.

6. Celera (2001), Celera Genomics, "http://www.celera.com/".

7. CO-IM (2001), Co-Operative Information Management Group, "http://www.science.uva.nl/ netpeer/".

8. DOE (2000), DOE2000 Collaboratories, "http://www-unix.mcs.anl.gov/DOE2000/collabs.html".

9. Foster, I. and Kesselman, C. (1998), The Globus Project: A Status Report., in proceedings of IPPS/SPDP ' 98 Heterogeneous Computing Workshop,

10. Kaletas, E. C., Afsarmanesh, H. and Hertzberger, L. O. (2001), Virtual Laboratory Experimentation Environment Data Model, University of Amsterdam, Informatics Institute, Amsterdam, Technical report CS-2001-01.

11. MGED (2001), MIAME - Minimum Information About a Microarray Experiment, "http://www.ebi.ac.uk/microarray/MGED/Annotations-wg/index.html".

12. Williams, G. (1999) Nucleic acid and protein sequence databases in Genetics Databases, (Ed, Bishop, M. J.) Academic Press, pp. 11-37.

\footnotetext{
i This work was supported by an ICES/KIS-II grant from the Dutch Ministry of Education and Sciences and the Ministry of Economic Affairs.
} 